$(\mathrm{aOR}=2.4,95 \% \mathrm{CI}=1.8-3.1$ for $\mathrm{SRH}$; $\mathrm{aOR}=3.0,95 \% \mathrm{CI}=2.0-$ 4.5 for $\mathrm{PHU})$, and client-related burnout $(\mathrm{aOR}=1.6,95 \%$ $\mathrm{CI}=1.2-2.3$ for $\mathrm{SRH}$; $\mathrm{aOR}=2.1,95 \% \mathrm{CI}=1.2-3.5$ for $\mathrm{PHU}$ ) while adjusted for significant variables.

Conclusions We concluded that nurses worked in public health units and secondary referral hospitals had higher job strain and work-related burnout as compared to primary clinics. Further study should examine the stressors from these workplaces and follow up the health effects of high strain and burnout status.

\section{METAGENOMIC DETECTION OF BACTERIA IN AEROSOL SAMPLES IN ANIMAL SLAUGHTERHOUSES TO DEVELOP EXPOSURE PROFILES FOR AN EPIDEMIOLOGICAL ANALYSIS}

${ }^{1}$ David McLean, ${ }^{2}$ Patrick Biggs, ${ }^{3}$ Mily Leblanc-Maridor, ${ }^{4}$ Richard Hall, ${ }^{2}$ Nigel French, ${ }^{5}$ Neil Pearce, 'Jeroen Douwes. ${ }^{1}$ Massey University, Wellington, New Zealand; ${ }^{2}$ Massey University, Palmerston North, New Zealand; ${ }^{3}$ Universite de Nantes, Nantes, France; ${ }^{4}$ Institute of Environmental Science and Research, Upper Hutt, New Zealand; ${ }^{5}$ London School of Hygiene and Tropical Medicine, London, UK

\subsection{6/oemed-2014-102362.65}

Objectives Significant excess risks of lung cancer and haematologic neoplasms have been observed in slaughterhouse workers in eight New Zealand studies, and numerous studies conducted elsewhere. No specific causal agents have been identified, although a biological aetiology is suggested as the risk is highest in those areas where workers are exposed to live animals or to biological material containing animal urine, faeces or blood. This study aimed to assess the airborne bacterial microflora in the slaughterhouse environment in order to develop exposure categories for reanalysis of a meat workers' cohort.

Method Bulk air samples $(\mathrm{n}=31)$ were collected for between 5 and $8 \mathrm{~h}$ in five areas in both sheep and beef slaughterhouses using a SASS3100 sampler (fitted with a proprietary SASS filter) located between 0.5 and 2 metres from the worker. Nucleic acid was extracted from each filter and amplified using commercially available kits, then sequenced on an Illumina MiSeq instrument. Bioinformatics analyses conducted included comparative taxonomic analyses, gene function (including virulence factor) analyses, and principal component analyses to compare profiles in samples taken in different areas.

Results Of the bacteria identified over 95\% were in the classes Actinobacteria, Firmicutes and Proteobacteria. Clear differences in all parameters were apparent in the different areas, however, and the full results of the comparative analyses and the development of exposure profiles will be presented.

Conclusions Metagenomic analysis of bioaerosol samples represents a promising method for the development of exposure categories for the epidemiological analysis of the effect of biological exposures in an occupational environment.

\section{SOMATISING TENDENCY, OCCUPATIONAL STRAIN AND MUSCULOSKELETAL SYMPTOMS: RESULTS FROM A LONGITUDINAL STUDY AMONG ITALIAN NURSES}

1,2Matteo Bonzini, 'Lorenza Bertù, ${ }^{2}$ Marco Conti, 'Alessia D'Amato, ' ${ }^{1}$ Giovanni Veronesi, ${ }^{3}$ David N Coggon, ${ }^{1,2}$ Marco M Ferrario. ${ }^{1}$ Epidemiology and Preventive Medicine Research Centre, Insubria University, Varese, Italy; ${ }^{2}$ MRC Lifecourse Epidemiology Unit, University of Southampton, Southampton, UK; ${ }^{3}$ Ospedale Di Circolo Fondazione Macchi, Varese, Italy

10.1136/oemed-2014-102362.66
Objectives Musculoskeletal symptoms are a common cause of disability, with major impact on workforce wellbeing, absenteeism and productivity. Several, mainly cross-sectional, studies have linked such symptoms to physical workload, and also to psychological and socio-cultural factors.

We investigated whether prolonged or increasing job strain, tendency to somatise and other individual characteristics, are associated with worsening musculoskeletal pain.

Method As part of the CUPID study, we investigated a cohort of nurses employed on medical wards at the Varese University Hospitals (Italy). Participants were asked, at baseline and after one year of follow-up, about individual and occupational risk factors, psychological characteristics (including tendency to somatise), occupational strain (by Siegrist's Effort/Reward Imbalance Questionnaire-ERI), and musculoskeletal symptoms. Associations of worsening musculoskeletal pain with perceived job strain were assessed by multivariate log-binomial regression.

Results Occupational stress was associated with pain in the lower back (LBP) and neck/shoulder (NSP) in both cross-sectional questionnaires.

Comparing baseline and follow-up answers, workers who reported an increase in perceived stress showed more frequent worsening of both LBP (prevalence of worsening symptoms $=41 \%$, OR when compared with not stressed $=1.7,95 \%$ $\mathrm{CI}=1.1-2.7$ ) and NSP (prevalence of worsening $=51 \%, \mathrm{OR}=1.2$, $95 \% \mathrm{CI}=0.8-1.8$ ).

This relationship persisted after adjustment for gender, age and BMI, and exposure to physical workload, and was more evident among subjects with a tendency to somatise $(\mathrm{OR}=2.8$. 95\% $\mathrm{CI}=1.0-7.4$ for $\mathrm{LBP}$; $\mathrm{OR}=1.6,95 \% \mathrm{CI}=0.8-3.2$ for NSP).

Conclusions Our observation suggests that tendency to somatise modifies individual responses to "triggering exposures", such as psychological workload, with important implications for the health, and productivity of workers.

\section{SINONASAL CANCERS: IS INTESTINAL TYPE ADENOCARCINOMA THE ONLY RELATED TO OCCUPATIONAL EXPOSURES? RESULTS FROM AN ITALIAN CASE-CONTROL STUDY}

${ }^{1,2}$ Matteo Bonzini, ${ }^{3}$ Laura Zanetta, ${ }^{1}$ Lorenza Bertù,
${ }^{3,4}$ Davide Parassoni, ${ }^{3}$ Mario Turri Zanoni,
Medicine Research Centre, Insubria University, Varese, Italy; ${ }^{2}$ Insubria University, Varese,
Italy; ${ }^{3}$ Ospedale Di Circolo, Fondazione Macchi, Varese, Italy; ${ }^{4}$ School of Specialisation in
Occupational Health, University of Brescia, Brescia, Italy

10.1136/oemed-2014-102362.67

Objectives Epithelial sinonasal cancers (SNC) are rare, severe diseases associated to the exposure to several well-established carcinogens (IARC). The etiologic role of these carcinogens in different histological subtypes is still disputed, with several studies focusing on intestinal-type adenocarcinoma (ITAC) as the most (and maybe the only) occupational-related subtype. To assess the role of occupational exposures in SNC aetiology we designed a case control study, in which occupational exposures prevalence in two group of ITAC cases and non-ITAC were compared to controls.

Method In a large Italian hospital we enrolled 50 consecutive surgical non-ITAC cases (mainly squamous-cell carcinoma), 50 consecutives ITAC cases and 50 non-neoplastic patients (controls). Previous occupational exposures to wood and leather dust, solvents, metals, formaldehyde were investigated through a 
standardised interview. Gender, age, residence area and smoking were collected as potential confounders. Multivariate logistic regression was applied.

Results Considering all tumours together, we observed large increased risks for wood exposure $(\mathrm{OR}=6.9,95 \% \mathrm{CI}=3.0-16.3)$ and leather (prevalence $24 \%$ in tumours, $0 \%$ among controls) only. Compared to controls, we observed an increased risk for wood exposure $[\mathrm{OR}=7.7(95 \% \mathrm{CI}=2.6-22.5)]$ in ITAC cases, but not in non-ITAC cases $[\mathrm{OR}=0.8(95 \% \mathrm{CI}=0.2-3.1)]$. Prevalence of leather exposure was $42 \%$ among ITAC and $6 \%$ in non-ITAC.

Conclusions Our case control study confirmed that ITAC cases but not other histotypes were strongly related to occupational exposures, and in particular to leather and wood dusts. Grouping together all SNC types reduce the causal role of occupation exposures. Larger samples size are needed to investigate other work-related carcinogens.

\section{DO FAMILY AND INDIVIDUAL CHARACTERISTICS AFFECT THE EXPERIENCE OF PHYSICAL AND PSYCHOSOCIAL WORK ENVIRONMENT IN DANISH 20/21 YEAR OLDS?}

${ }^{1}$ Trine Nohr Winding, ${ }^{1,2}$ Merete Labriola, ${ }^{3,4}{ }^{2}$ Ellen Aagaard Nohr, ${ }^{1}$ Johan Hviid Andersen. ${ }^{1}$ Danish Ramazzini Centre, Department of Occupational Medicine, Regional Hospital Herning, Herning, Denmark; ${ }^{2}$ Department of Clinical Social Medicine, Public Health and Quality Management, Central Denmark Region and Section of Clinical Social Med, and Rehabilitation, School of Public Health, Aarhus University, Aarhus, Denmark; ${ }^{3}$ University of Southern Denmark, Institute of Clinical Research, Research Unit of Gynaecology and Obstetrics, Odense, Denmark; ${ }^{4}$ Department of Public Health, Section for Epidemiology, Aarhus University, Aarhus, Denmark

\subsection{6/oemed-2014-102362.68}

Objectives To describe the work environment of Danish 20/21 year olds and to investigate the influence of family socioeconomic background or individual characteristics at age $14 / 15$ on later experience of physical and psychosocial work environment.

Method The study population consisted of 695 young people with primary work affiliation at age $20 / 21$ who were derived from a prospective youth cohort. Outcome information from the questionnaire in 2010 consisted of six questions about psychosocial work environment and two questions about physical work environment. Exposure information about school performance, vulnerability, health and parental socioeconomic status was derived from the questionnaire in 2004 and from registers.

Results Overall, the psychosocial work environment of the young people was good but they experienced more repetitive movements and hard physical work than older workers. Individual as well as family factors in late childhood all together only had limited impact on how young people report later work environment. Low self-esteem at age 14/15 was associated with experiencing high demands, low trust and low fairness at work. In girls low self-esteem and low sense of meaningfulness were associated with experiencing low influence at work. Low parental socioeconomic status was associated with poor physical work environment.

Conclusions This study showed a social gradient in experiencing poor physical work environment at age 20/21. The psychosocial work environment in young people was on average good, but it seems that vulnerable young people need special intention in order to prevent them from being selected into psychosocial demanding job functions later in life.

\section{3}

GROUPING STRATEGIES FOR EXPOSURE ASSESSMENT OF THE PSYCHOSOCIAL WORK ENVIRONMENT

${ }^{1}$ Morten Vejs Willert, ${ }^{1,2}$ Vivi Schlünssen, ${ }^{2}$ loannis Basinas, ${ }^{1}$ Zara Ann Stokholm, ${ }^{3}$ Matias Brødsgaard Grynderup, ${ }^{4}$ Johan Hviid Andersen, ${ }^{5}$ Reiner Rugulies, ${ }^{3}$ Åse Marie Hansen, ${ }^{6}$ Linda Kærlev, ${ }^{7}$ Jane Frolund Thomsen, ${ }^{7}$ Marianne Agergaard Vammen, ${ }^{1}$ Henrik Kolstad. ${ }^{1}$ Danish Ramazzini Centre, Department of Occupational Medicine, Aarhus University Hospital, Region Midt, Denmark; ${ }^{2}$ Danish Ramazzini Centre, Department of Occupational Medicine, Herning University Hospital, Region Midt, Denmark; ${ }^{3}$ Research Unit of Clinical Epidemiology, Institute of Clinical Research, University of Southern Denmark, Region Southern Denmark, Denmark; ${ }^{4}$ Department of Public Health, Aarhus University, Aarhus, Denmark; ${ }^{5}$ Department of Public Health, University of Copenhagen, Copenhagen, Denmark; ${ }^{6}$ Research Centre for the Working Environment, Copenhagen, Denmark; 'Department of Occupational and Environmental Medicine, Bispebjerg University Hospital, Capital Region, Denmark

\subsection{6/oemed-2014-102362.69}

Objectives Individual response style, mood, expectations, and health status may affect reporting of the psychosocial work environment, and bias associations with outcomes. Reporting bias may be avoided by aggregating individual responses, ideally preserving exposure contrast. In this study, we examined the degree of exposure contrast yielded by different grouping strategies. Method In 2007, we enrolled 4489 public employees from Aarhus, Denmark in the PRISME-cohort, with follow-up in 2009. From pay-roll registers we grouped workers at 2 organisational levels: department $(\mathrm{n}=22)$ and work unit $(\mathrm{n}=751)$, and 3 occupational levels: sector $(n=7)$, profession $(n=46)$, and job title $(\mathrm{n}=77)$. Exposures, calculated as means of items scored on 5 -point Likert scales, included psychological demands, decision latitude, social support, effort, reward, and procedural and relational justice. To assess variance components, we fitted linear mixed effect models with exposures as dependent variables, and id and grouping variables as random effects. Results are reported as the contrast in mean exposure levels e.g. between-group variance/ (between-group variance + within-group variance).

Results Within each hierarchy contrasts rose with increasing grouplevel detail. Grouping by either work unit (wu) or by job title (jt) contrasts were: psychological demands: $0.28(\mathrm{wu}) ; 0.26(\mathrm{jt})$, decision latitude: $0.24(\mathrm{wu}) ; 0.32(\mathrm{jt})$, social support: $0.24(\mathrm{wu}) ; 0.06(\mathrm{jt})$, effort: $0.23(\mathrm{wu}) ; 0.16(\mathrm{jt})$, reward: $0.19(\mathrm{wu}) ; 0.12(\mathrm{jt})$, procedural justice: $0.24(\mathrm{wu}) ; 0.14(\mathrm{jt})$, and relational justice: $0.29(\mathrm{wu}) ; 0.04(\mathrm{jt})$. Conclusions Grouping by work unit gave the most consistent contrasts $(0.19-0.29)$, while grouping by job title varied considerably (0.04-0.32). These preliminary findings suggest that grouping by work unit provided better exposure contrasts than grouping by job title for all exposures, but decision latitude.

\section{PREDICTORS OF WORKERS' COMPENSATION CLAIM DURATION AMONG WORKERS DISABLED DUE TO LOW BACK PAIN}

${ }^{1}$ Jason Busse, ${ }^{2}$ Ivan Steenstra, 'Shanil Ebrahim, 'Diane Heels-Ansdell, 'Stephen Walter, ${ }^{1}$ Gordon Guyatt. ${ }^{1}$ McMaster University, Hamilton, Ontario, Canada, ${ }^{2}$ Institute for Work and Health, Toronto, Ontario, Canada

\subsection{6/oemed-2014-102362.70}

Objectives Low back pain (LBP) is a common complaint among workers receiving Workers' Compensation wage replacement benefits. We used the administrative data from the Ontario Workplace Safety and Insurance Board (WSIB) to explore the association between baseline characteristics and commonly reimbursed therapies and time to claim closure among workers disabled due to LBP. 